\title{
The effect of physical factors on the vascular endothelium in children with primary arterial hypertension
}

\author{
Yanina Tetyana , Kaladze Nikolay \\ Republic Children Clinical Hospital, V. I. Vernadsky Crimean Federal University \\ Simferopol, Russia
}

\section{Background and Aims}

Early diagnostics of arterial hypertension (HTN) for children, design the hypertension prophylaxis program are an important scientific and pract ical problem. The aim of our research was optimization of rehabilitation measures on the resort stage for children with HTN on studying and then correction the functional state of vascular wall endothelia. Researches were conducted on the resort bases in Crimea during 2011-2017.

Method: In our tasks included: to study to the feature of day's blood pressure profile for children with essential HTN; to investigate the morphological and functional state of heart, to find the features of speed indexes of blood stream and complex of intima-media of general carotids for children with $\mathrm{EAH}$; to estimate the vasoregulation function of endothelia vessels for children with essential HTN; to conduct the complex estimation biochemical markers of the cond ition endothelia vessels function (total metabolites of oxide nitrogen (NO), endothelin-I (ET-I), asymmetric dymethilarchinin (ADMA) Also we conducted the comparative estimation of efficiency of the use in the rehabilitation of children with essential HTN of CL-Na baths from the natural thermal sources from Crimea resort in a complex from a neurofeedback, for educating of children with EAT $t$ o skills of relaxation, for normalization of neurohumoral mechanisms of adjusting

Results: 114 children are inspected with essential HTN in age 11-16 (middle

age

$13,8 \pm 0,2)$. At an inspection the row of exogenous factors was educed: $10,5 \% \quad$ children - smoked; 45,6\% - preferred the fast food, fried and sharp food; $18,4 \%$ - have predilection to strongly salt food, and $11,4 \%$ additionally add some salt already the prepared food. The standard complex of resort treatment included: the mode of day with a night sleep no less than 9 hours; limitation of gadgets, protracted walks and sp orting games on fresh air, command sporting games; valuable balanced feed (with reduction of salt consumption, reduction of carbohydrate ration), morning gymnastic, massage, school on propaganda of healthy lifestyle.

\section{Conclusion}

Combination traditional resort treatment with method of neurofeedback and CL-Na baths from natural thermal sources

improve

quality of life of children due to a positive dynamics on complaints, for certain $(p<0,01)$ to bring down average daily indexes BP, positively to influence on the state of function of endothelia, bring $(p<0,01)$ down the levels of ET-I and ADMA on a background the increase of NO maintenance.
Cathamnesis supervision results for children with HTN a tendency is reduced the therapeutic effect and growth of disbalance endothelial markers after 52 weeks, that explained by the interruption between the sanatoriumresort stages of rehabilitation.
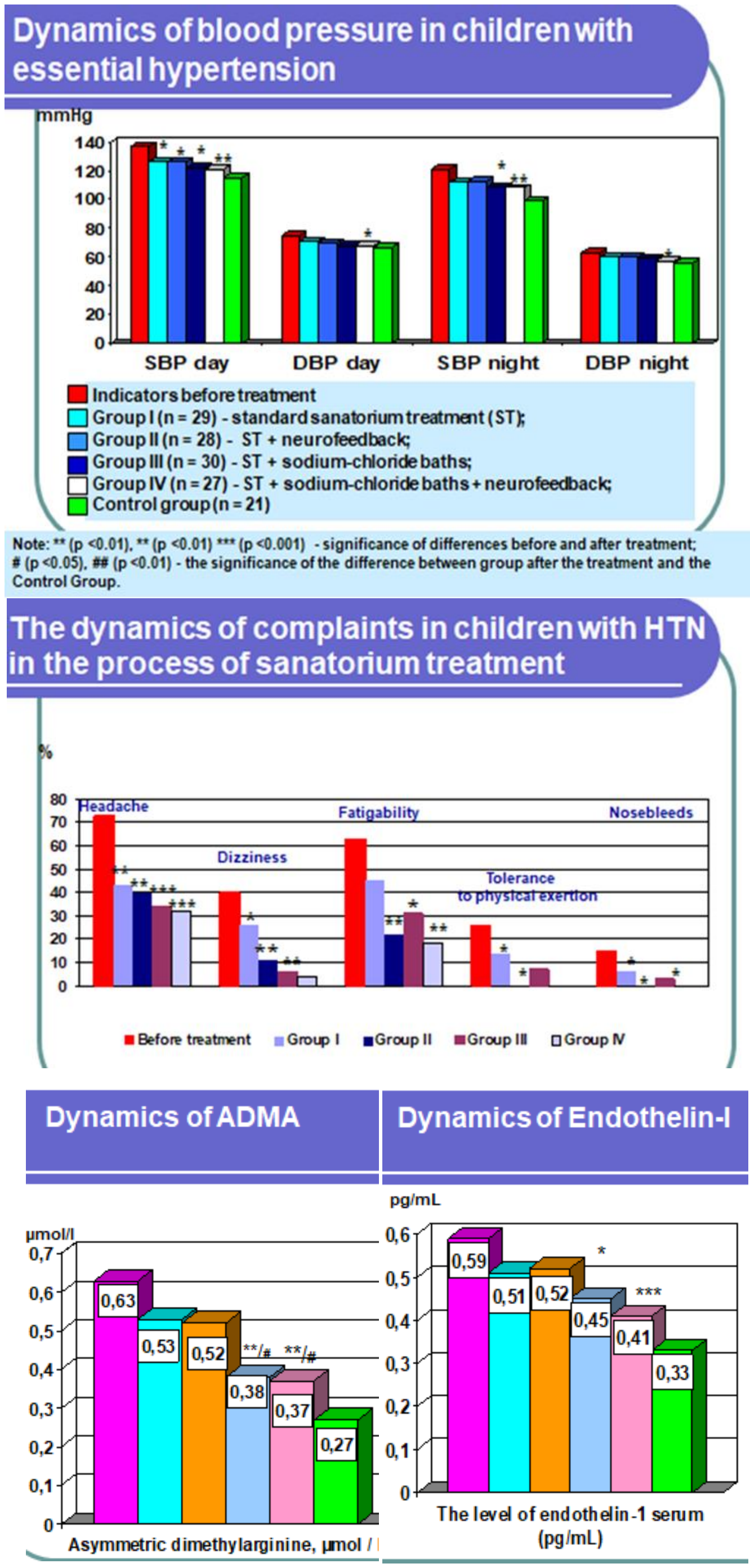

Dynamics of NO levels

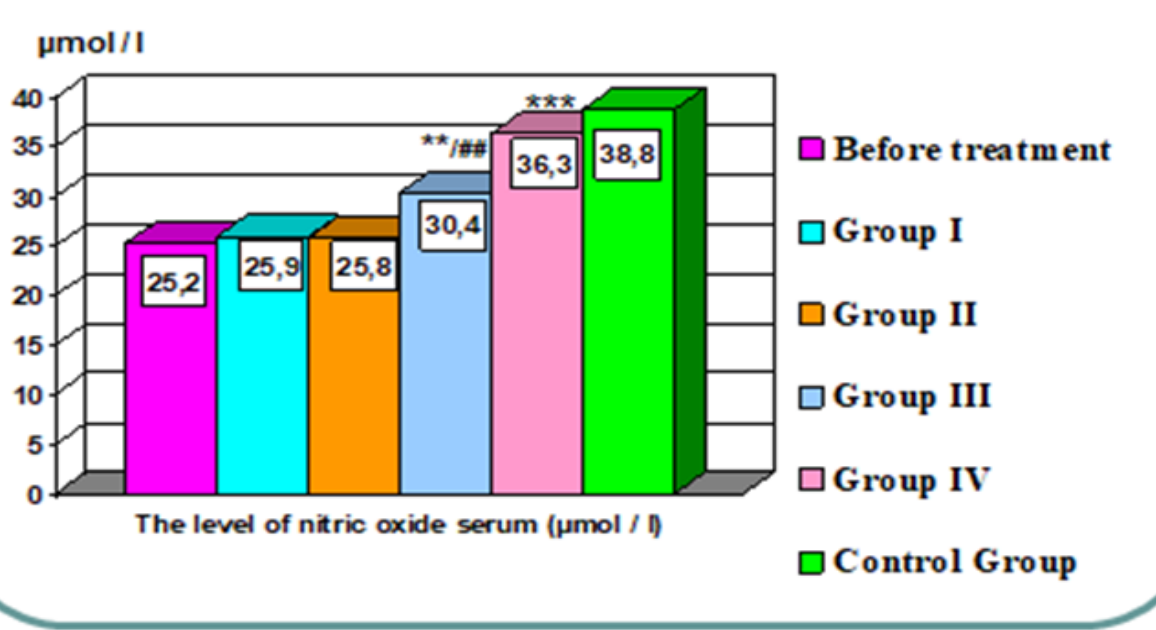

